\title{
The design of dummy ROV electric-drive seven functions manipulator
}

\author{
Zaiyu Chen ${ }^{1,}$, Meina Guo ${ }^{1, b}$, Zhenzhong Liu ${ }^{2}$ and Lianyu Zhao ${ }^{2}$ \\ ${ }^{1}$ Offshore Oil Engineering Co., Ltd. Tianjin, China; \\ ${ }^{2}$ Tianjin Key Laboratory of the Design and Intelligent Control of the Advanced Mechatronical System, \\ Tianjin University of Technology, Tianjin, China. \\ achzy@mail.cooec.com.cn, bguomn@mail.cooec.com.cn.
}

Keywords: Dummy ROV, seven functions manipulator, manipulator design.

\begin{abstract}
With the deepening of the Marine development, underwater constructing often need to use the ROV. In order to verify the construction scheme, ensuring construction efficiency, the staff will use ROV or Dummy ROV on land to simulate construction. As the main body of the ROV, the manipulator is the execution part of the system. It is the core of the whole system; all of the drive and control are around it. It references the mature underwater manipulator, and draw lessons from the domestic manipulator design experience. The design adopts electric drive, and the joints torque were calculated and simulated by the SolidWorks motion analysis software.
\end{abstract}

\section{Introduction}

All manuscripts must be in English, also the table and figure texts, otherwise we cannot publish your paper. Please keep a second copy of your manuscript in your office. When receiving the paper, we assume that the corresponding authors grant us the copyright to use the paper for the book or journal in question. Should authors use tables or figures from other Publications, they must ask the corresponding publishers to grant them the right to publish this material in their paper. Use italic for emphasizing a word or phrase. Do not use boldface typing or capital letters except for section headings (cf. remarks on section headings, below).

Do not number your paper: All manuscripts must be in English, also the table and figure texts, otherwise we cannot publish your paper. Please keep a second copy of your manuscript in your office. When receiving the paper, we assume that the corresponding authors grant us the copyright to use the paper for the book or journal in question.

\section{Seven function manipulator overall design}

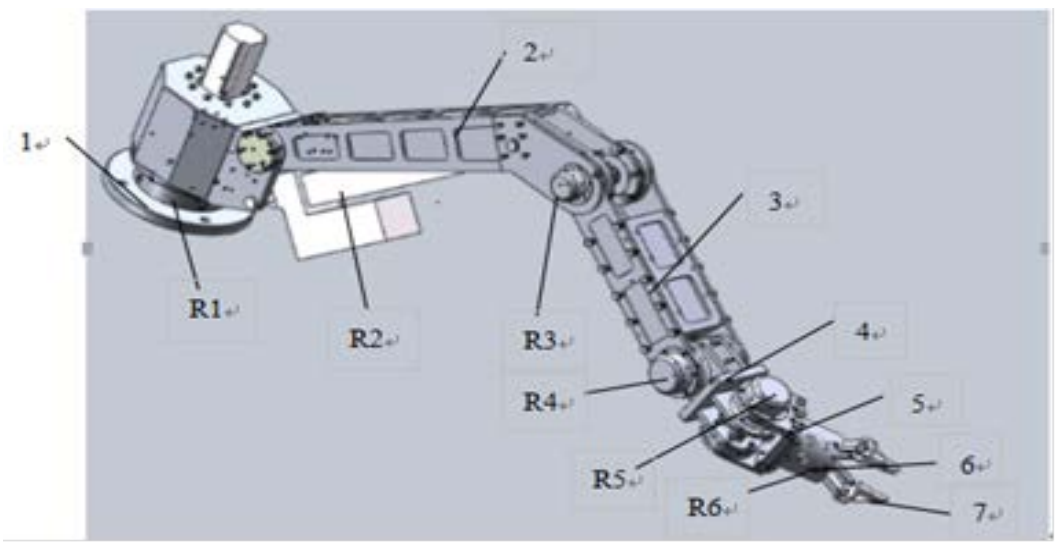

1: base, 2: big arm, 3: big arm, 4: wrist, 5: wrist, 6: wrist, 7: hand grasp

R1: shoulder swings around the joints R2: big arm pitching movement joints

R3: elbow pitch movement joints

R4: wrist pitch movement joints

R5: wrist swinging movement joints R6: wrist rotation movement joints

Fig. 1 Seven functions manipulator structure diagram 
Manipulator is a central part of the whole manipulator system and its structure as shown in figure 1. It is powered by a $220 \mathrm{VAC}$ power and servo motor and electric draw stem or high precision harmonic reducer drive, to achieve the six joint movement and the clutches opening and closing of swing, big arm pitching, elbow pitch, forearm, wrist pitch, wrist turned.

\section{Seven function of mechanical structure design of wrist and elbow}

\subsection{The driver module design}

The design of the driver module can be divided into two kinds. The first is separate design of the motor, reducer, drive, and brake. And the second is integrated design of the motor, reducer, drive, and brake. Separate design transmit by synchronous belt, possesses the advantages of fast response. But compared with the integrated short transmission chain of large space, the structure is compact, low transmission efficiency, become the inevitable shortcomings. In this paper, according to the need to consult integration module to integrate the (1) encoder, (2) motor, (3) gear reducer, (4) brake, etc in a standard module, used in the machine arm forearm pitch, wrist pitch and swinging, greatly reduces the repetitive design work, reduce the volume of a module, and realize the mechanical arm structure compact.

\subsection{The connection module design}

To connect the driver module and form the whole mechanical arm, big arm, forearm, wrist connecting rod structure are according to each driver module type mechanical the physical size of the interface and determine the length of each part of the bar. Considering of the beautiful of mechanical arm and the flexibility of movement, as well as the internal line convenience, each connection module with hollow hole, each module of the power line and data line can be an internal line through module hollow shaft hole and connecting rod hollow hole. Mechanical arm configuration requirements of the forearm and wrist can realize the rotary motion, thus the forearm and wrist designed by rotary module connection. Each connection module structure is shown in figure 2 and figure 3.

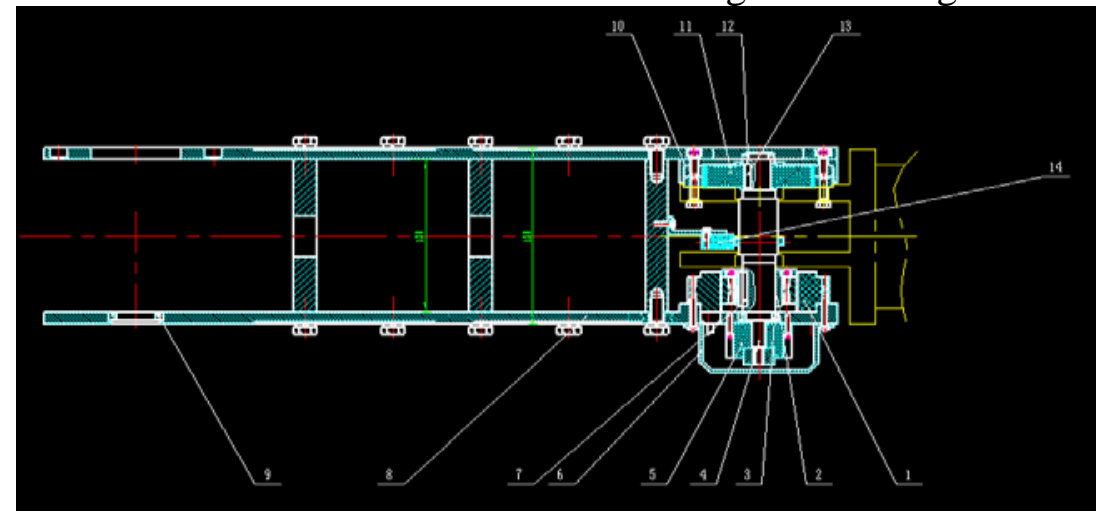

Fig. 2 Wrist pitch structure design

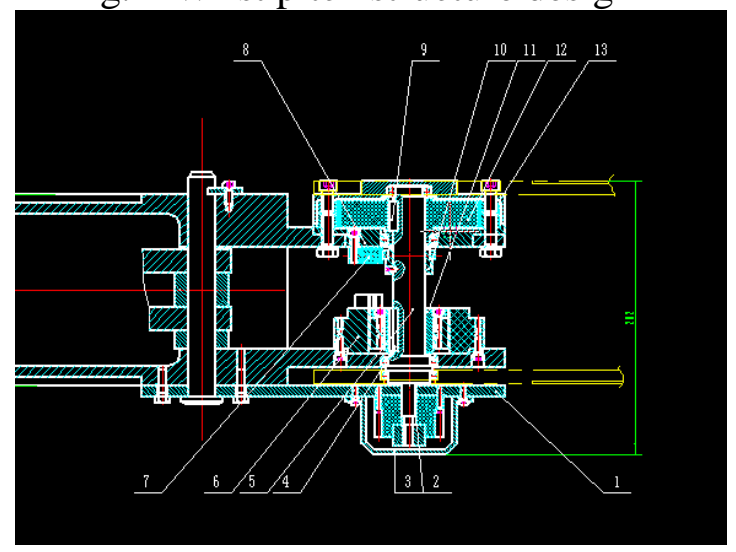

Fig. 3 Forearm pitch design

\subsection{The manipulator arm pitching design}

Under the condition of adapt to the working environment, the big arm mainly afford four aspects of 
the external force such as under gravity of its own ,mechanical arm of the forearm, wrist, clutches, motor and reducer on each joint and weight of clutches. More easy to cause deformation of slender structure big arm force, and simply rely on motor and speed reducer is very difficult to achieve large arm pitching in small space movement, considering the above factors to select motor cylinder to drive, drive structure as shown in figure 4.

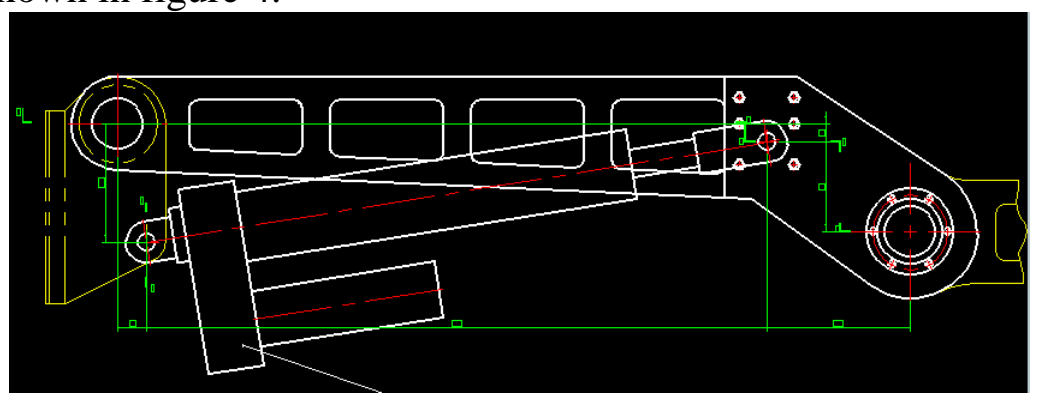

Fig.4 The big arm pitching design

\subsection{The manipulator large arm rotate design}

Base is the basis of the mechanical arm part of the mechanical arm, playing a supporting role, directly fixed on the steel structure framework, operation in the process of the force transmitted to the base of other parts, so as to prevent deformation of base, base wall thickness design for $15 \mathrm{~mm}$, inner sleeve as the main supporting parts selecting 45 \# steel material. During operations, base need to implement large arm swinging function, so the mechanical arm driver implementation needs the collocation of servo motor and reducer. As shown in figure 5 servo motor output shaft with harmonic reducer coupling wave generator, soft round of harmonic gear reducer through combined with inner sleeve fixed on the base, power through the first round of the output speed reducer, drive shell or arm can rotate on the support plate.

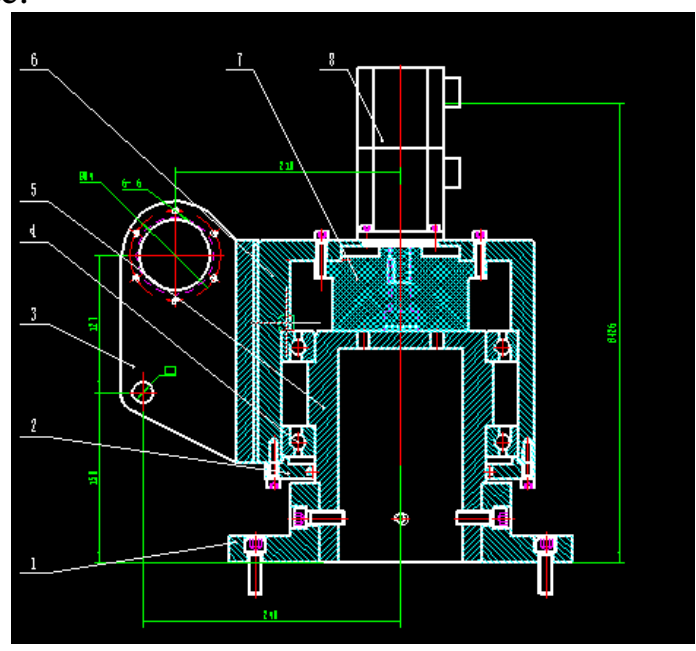

Fig. 5 The large arm rotation design

\section{Seven function manipulator joint torque calculation}

\subsection{Seven function manipulator gripper calculation}

(1) Hand grasp force calculation:

Hand grasp on the workpiece on the tightening force is the main basis of design of the hand, must analyze its size, direction and functional point to calculation. In general, tightening force has to overcome the gravity of the artifacts produced by static load (inertial force and inertial moment) to make the workpiece will remain reliable stepped up.

Hand grasp tightening force of artifacts may refer to the formula to calculate:

$$
F_{N} \geq K_{1} K_{2} K_{3} G
$$

Type: 
$K_{1}$----Safety factor, Be determined by the process and the design requirements of the manipulator, usually from 1.2 to 2.0 , here take 1.5 ;

$K_{2}$----Coefficient of performance, Mainly consider the effect of inertia force, the calculation of maximum acceleration, coefficient of performance $K_{2}, K_{2}=1+a / g$. $a$ for the mechanical arm carrying workpieces in the process of the absolute value of the acceleration or deceleration, take $K_{2}=1_{\text {here; }}$

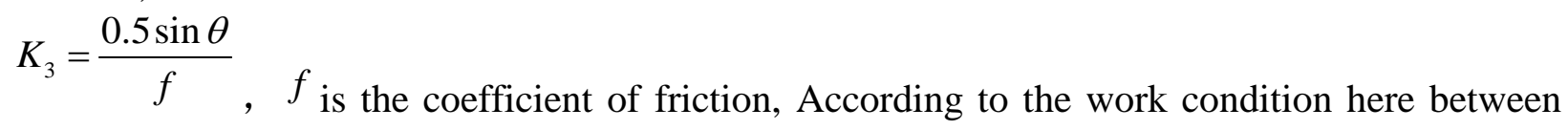
aluminum alloy friction, take $f=0.5$ here;

To sum up, force calculation hand grasp:

$$
F_{N} \geq K_{1} K_{2} K_{3} G=1.5 \times 1 \times 0.7 \times 80=84 N \text {. }
$$

Inertia:

$$
\begin{array}{r}
J=\frac{1}{2} m R^{2}=0.024625 \mathrm{~kg} \cdot \mathrm{m}^{2} \\
M=J \alpha=0.24625 \mathrm{NM}
\end{array}
$$

$0.5 \mathrm{NM}$.

\subsection{Seven function manipulator wrist pitch calculation}

In the system, the motor rotation as the drive source of a manipulator, it must overcome the mechanical arm system work heavy torque and the maximum torque caused by motor acceleration. Due to the size of wrist pitch and wrist swinging space and form is consistent, so the wrist swinging motor and wrist pitch model is possible consistent, the result will only appear the phenomenon of " big horse-drawn car ", can achieve the engineering requirement. To choose the suitable motor, first of all to realize wrist pitch motor output torque calculation, the calculation process is as follows:

To achieve wrist pitch, firstly to overcome the motor output shaft torque by the swing arm forearm and wrist joints, and the clamping manipulator claw weight of gravity, according to the law of gravitational torque heavy torque can be obtained.

$$
M_{1}=\mathrm{mgl}_{c}=250 \times 0.38=95 \mathrm{NM}
$$

In the process of pitch, mechanical arm joint wrist need to experience starting from 0 up to the required angular velocity, then the uniform rotation. In the process of from static acceleration speed torque is produced. Required mechanical arm speed $10 \mathrm{r} / \mathrm{min}$, which is $1 \mathrm{rad} / \mathrm{s}$.

$$
\begin{gathered}
\left(3600^{\circ} / 180\right) \times 3.14 / 60=1 \mathrm{rad} / \mathrm{s} \\
\frac{1}{2} \alpha t^{2}=\omega=1 \mathrm{rad} / \mathrm{s} \\
\alpha=2 \mathrm{rad} / \mathrm{s}^{2}
\end{gathered}
$$

From the moment of momentum theorem and formula we can obtain the wrist pitch motion acceleration torque.

$$
M_{2}=J \alpha=\frac{1}{3} m\left(2 l_{c}\right)^{2} \alpha=\frac{4}{3} \times 25 \times 0.38^{2} \alpha=4.82 \alpha=9.64
$$

The output torque of the motor decided jointly by the heavy torque and acceleration torque, so the total output torque is as the follows 


$$
M_{\text {总 }}=M_{1}+M_{2}=105 \mathrm{NM}
$$

\subsection{Seven function manipulator elbow pitch calculation}

Elbow in driven by motor as the power source of the forearm pitch, same here in order to achieve lower pitch it also need to overcome heavy torque of mechanical arm system work; motor acceleration caused by maximum torque. Through 3D software localization, choose wrist rotation body center as centroid, $572 \mathrm{~mm}$ from the big arm shaft.

Firstly to overcome the motor output shaft torque by the swing arm forearm and wrist joints, and the clamping manipulator claw weight of gravity, according to the law of gravitational torque heavy torque can be obtained.

$$
M_{1}=\mathrm{mgl}_{c}=377.68 \times 0.642=242.5 \mathrm{NM}
$$

In the same process of mechanical arm pitch, forearm joint forearm need to experience starting from 0 up to the required angular velocity, then the uniform rotation. In the process of from static acceleration speed torque is produced. Motion of manipulator 270 degree also 4.71 rad, mechanical arm speed required $10 \mathrm{r} / \mathrm{min}$, which is $1 \mathrm{rad} / \mathrm{s}$.

$$
\begin{aligned}
\frac{1}{2} \alpha t^{2} & =\omega=1 \mathrm{rad} / \mathrm{s} \\
\alpha & =2 \mathrm{rad} / \mathrm{s}^{2}
\end{aligned}
$$

From the moment of momentum theorem and formula we can obtain the wrist pitch motion acceleration torque.

$$
M_{2}=J \alpha=\frac{1}{3} m\left(2 l_{c}\right)^{2} \alpha=\frac{4}{3} \times 37.768 \times 0.642^{2} \alpha=20.75547994 \alpha=42
$$

The output torque of the motor decided jointly by the heavy torque and acceleration torque, so the total output torque is as the follows

$$
M_{\text {总 }}=M_{1}+M_{2}=284.5 N M
$$

\subsection{The calculation of seven function manipulator arm can pitch}

The place of elbow also driven by servo electric cylinder that the power source. Here to achieve lower arm pitch so that it also needs to overcome the gravity of the mechanical arm in the process of system work and the inertia in the process of swing. Through 3D software localization, choose wrist rotation center as the center of mass.

Firstly to overcome the gravity by the mechanical arm, the manipulator claw and the thing of holding. In the process of mechanical arm joint pitch, to overcome the heavy torque of 666.69 NM and arms need to experience starting from 0 up to the required angular velocity, then the uniform rotation. In the process of from static acceleration speed torque is produced. Motion of manipulator 120 degree also $2.09 \mathrm{rad}$, mechanical arm speed required $10 \mathrm{r} / \mathrm{min}$, which is $1 \mathrm{rad} / \mathrm{s}$.

$$
\begin{gathered}
\frac{1}{2} \alpha t^{2}=\omega=1 \mathrm{rad} / \mathrm{s} \\
\alpha=2 \mathrm{rad} / \mathrm{s}^{2}
\end{gathered}
$$

The dynamic torque of big arm is:

$$
M=J \alpha=28.89 N M
$$

The total torque for big arm need is:

$$
M_{\text {总 }}=M_{\text {重 }}+M=695.58 N M
$$

After the conversion of the torque and thrust, electric cylinder thrust required for $5649.53271 \mathrm{~N}$. 


\subsection{The calculation of seven function manipulator big arm swinging.}

Base in the same driven by motor that the power source of the big arm swinging, here to achieve big arm swinging need to overcome the system work motor acceleration caused by the maximum torque.

Large mechanical arm swinging arm joint process, big arms need to experience starting from 0 up to the required angular velocity, then the uniform rotation. In the process of from static acceleration speed torque is produced. Required mechanical arm speed 10s for a circle and $1 \mathrm{~s}$ acceleration, $1 \mathrm{~s}$ to slow down.

$$
\begin{gathered}
J=\frac{1}{2} m R^{2}=164.929 \mathrm{~kg} \cdot \mathrm{m}^{2} \\
M=J \alpha=115.45 \mathrm{NM}
\end{gathered}
$$

\section{The movement simulation of seven functional mechanical arm}

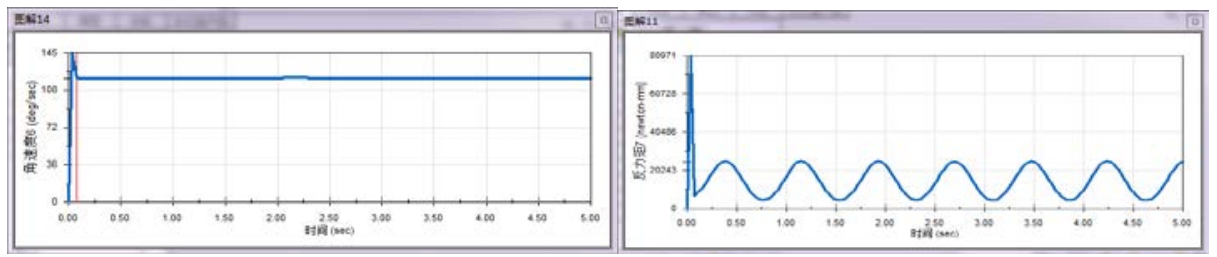

Fig. 6 The wrist joint angular velocity

Fig. 7 The wrist joint torque

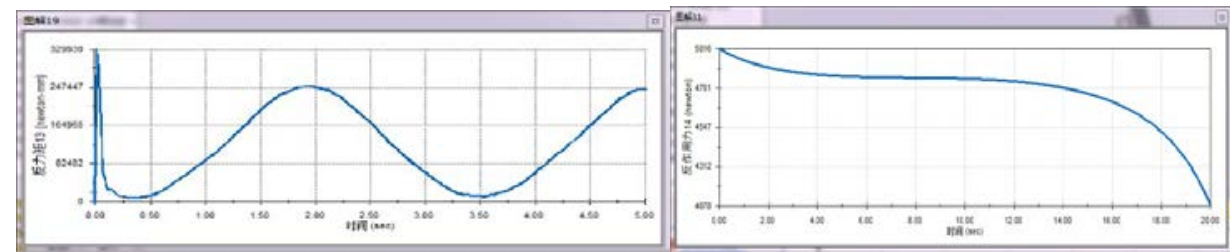

Fig. 8 The elbow torque

Fig.9 The big arm thrust

Under the premise of meeting the connection between the parts and the requirement of the range of movement, mechanical arm motion simulation add the corresponding motor drive, the simulation analysis was carried out on the design of the mechanical arm movement. Using SolidWorks motion function, make the trajectory simulation of the mechanical arm to obtain robotic arm torque of each joint.

(l)Forearm wrist movement

Firstly, sets the forearm to the fixed, add wrist rotation motor, so as to realize the movement of the wrist and hand. Elbow wrist pitching Angle displacement plus or minus 90 degrees Fahrenheit for lower arm, add gravity to determine the wrist pitch direction for the direction of gravity. Starting from the initial position, forearm to movement according to the set of angular velocity and angular position. After the simulation, SolidWorks automatically generate the torque curve as shown in figure 5-7.

(2)Big arm's elbow motion

Set the big arm in fixed, forearm, wrist and gripper in floated, add the motor of phase rotation for the big arm joint velocity of $20 \mathrm{RMP}$, determine the direction of gravity at the same time, simulation with elbow torque as shown in figure 5-8.

By means of the simulation results compared with the theoretical calculation, we found that the results of theoretical calculation is slightly superior to the results of simulation, mainly due to the safety factor in the process of theoretical calculation is slightly larger, at the same time, considering the engineering practice in the process of calculation, the phenomenon of the "big horse-drawn car" rather than to ensure that the structure is normal and safe and reliable operation, so the center of mass for arm and parts quality estimate is conservative in the process. But the simulation results show that: The motor and speed reducer be selected according to the theoretical calculation value can guarantee 
the normal work of the institutions, verified the validity of the calculation. The dummy ROV is shown in the figure 10.

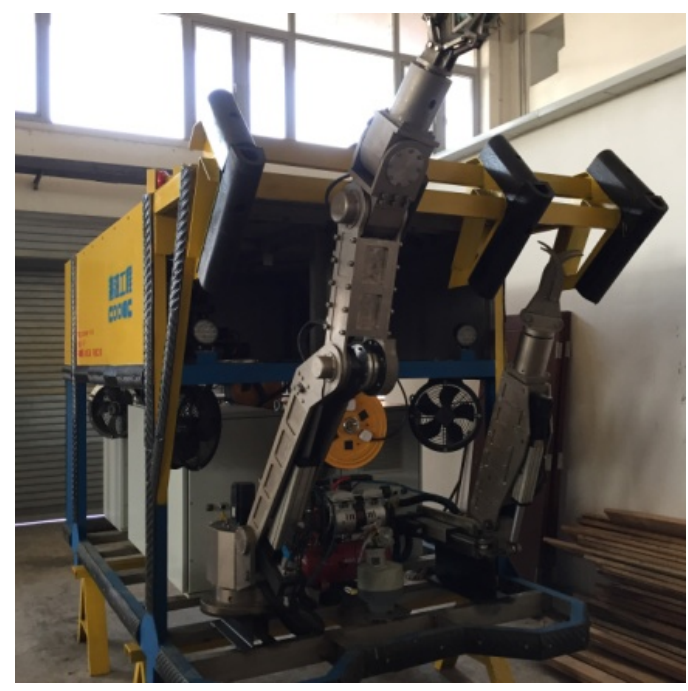

Fig. 9The dummy ROV

\section{Summary}

In this paper, the Dummy ROV seven functions manipulator was carried on the structure design, torque of each joint is calculated and using SolidWorks software of motion function for each joint motion simulation analysis. According to actual condition to make each joint to uniform motion and mainly analyzes whether the output torque of each joint is consistent with the calculated value. The results show that 1 ) Each joint torque calculation is correct, the simulation results are in conformity with the theoretical calculation results. 2) Mechanical structure design is reasonable, under the drive torque, smooth operation, stable output torque change, no interference in the process of the whole movement, comply with the design requirements. 3) The whole design of manipulator claw can provide basis for the mechanical processing and purchasing motor.

\section{References}

[1]. Hosseini M, Seyedtabaii S. Robust ROV path following considering disturbance and measurement error using data fusion. Applied Ocean Research. Vol. 54 (2016), p. 67-72.

[2]. Chowdhury T, Sathianarayanan D, Dharani G, et al. Failure Analysis of Fasteners in a Remotely Operated Vehicle (ROV) System. Journal of Failure Analysis and Prevention. Vol. 15(2015), No. 6, p.915-923.

[3]. Jin Sangrok, Kim Jihoon, Kim Jongwon, et al. Six-Degree-of-Freedom Hovering Control of an Underwater Robotic Platform with Four Tilting Thrusters via Selective Switching Control. IEEE/ASME Transactions on Mechatronics. Vol. 20(2015), No. 5, p. 2370-2378.

[4]. Maalouf Divine, Creuze Vincent, Chemori Ahmed, et al. Real-time experimental comparison of two depth control schemes for underwater vehicles. International Journal of Advanced Robotic Systems. Vol.12 (2015). 